\title{
Impact of vaccination against varicella on the reduction of the disease incidence in children and adolescents from Florianópolis, Brazil
}

\author{
Emil Kupek, ${ }^{1}$ Elaine F. Tritany ${ }^{2}$
}

\begin{abstract}
Objective: To evaluate the impact of universal early childhood vaccination against varicella on the reduction of its incidence in a state capital in southern Brazil.

Methods: Official epidemiologic surveillance data from the SINAN database for the 1997-2007 period were used to evaluate the impact of varicella vaccination targeting all children $<2$ years of age in Florianópolis (the capital city of the state of Santa Catarina), Brazil, since 2002, thus comprising 5 years before and 6 years after the vaccination. Varicella incidence in Florianópolis was compared with the incidence in the rest of the state for four age groups $(<1$, 1-4, 5-9, and 10-14 years).

Results: Among the 135,311 cases of varicella in the state of Santa Catarina during the 1997-2007 period, $70 \%$ were children under 10 years of age. The effectiveness of varicella vaccine ranged from 27 to $38 \%$ among the age groups but reached statistical significance only for children 1-4 years old.
\end{abstract}

Conclusion: The vaccine was effective in reducing varicella incidence in Florianópolis for children 1-4 years old.

J Pediatr (Rio J). 2009;85(4):365-368: Varicella, vaccine, effectiveness.

\section{Introduction}

In the Brazilian public health system, the vaccine against the varicella zoster virus (VZV) is mainly recommended for susceptible individuals with increased risk of a severe form of the disease or its complications, as well as for health professionals, family members or immunocompromised individuals in contact with an infected person. ${ }^{1}$ The vaccine is also available in private institutions throughout the country.

A recent analysis of cost-effectiveness of universal childhood vaccination in Brazil showed that a single dose of vaccine would be cost-effective under the assumption of $85 \%$ of vaccine efficacy and $80 \%$ of vaccine coverage. ${ }^{2}$ The hypothetical beneficial effects include prevention of over
74,000 cases and 2,900 deaths, as well as considerable reductions in hospitalizations ( $83 \%$ ) and in the number of outpatient healthcare services ( $85 \%$ ) due to primary VZV infection for the first 30 years of vaccine implementation. However, no empirical data on the impact of vaccination against VZV in Brazil have been published so far.

The municipality of Florianópolis, the capital city of the state of Santa Catarina, is the only place in Brazil where all children under 2 years of age have been targeted by the vaccine since 2002 , thus providing a unique opportunity to evaluate the vaccine effectiveness in reducing the disease incidence since then. The objective of this study is to provide the first results of such evaluation based on the comparison

1. Doutor, Departamento de Saúde Pública, Universidade Federal de Santa Catarina (UFSC), Florianópolis, SC, Brazil.

2. Especialista, Saúde Pública. Diretoria, Vigilância Epidemiológica, Secretaria de Saúde do Estado de Santa Catarina, Florianópolis, SC, Brazil.

The present study was carried out at Universidade Federal de Santa Catarina (UFSC), Florianópolis, SC, Brazil.

No conflicts of interest declared concerning the publication of this article.

Suggested citation: Kupek E, Tritany EF. Impact of vaccination against varicella on the reduction of the disease incidence in children and adolescents from Florianópolis, Brazil. J Pediatr (Rio J). 2009;85(4):365-368.

Manuscript submitted Dec 03 2008, accepted for publication Apr 012009

doi:10.2223/JPED.1909 
of the reported VZV incidence in the state capital and the rest of the state for an 11-year period, using the first 5 years in the pre-vaccination period as the reference.

\section{Methods}

We used the official epidemiological surveillance data for VZV infection (Sistema de Informação de Agravos de Notificação, SINAN) in the state of Santa Catarina during the period 1997-2007 and population statistics ${ }^{3}$ to calculate annual incidence rates for the state capital and the rest of the state. This is an ecological study comparing the incidence rate variation between these two areas using retrospective data collection.

Although individual notification of VZV infection is not compulsory in the whole country, except in the case of outbreaks, it has been introduced in Santa Catarina since 1994, leading to investigation of the notified individuals according to the guidelines of the Brazilian Ministry of Health. ${ }^{4}$ Case definition followed the guidelines and included those confirmed by laboratory, by signs and symptoms compatible with varicella, by epidemiological link to a confirmed case or any combination of these criteria. Most of the cases were diagnosed by maculo-papulovesicular rash without another apparent cause and persisting for more than 24 hours.

Since the year 2002, all children up to the age of 2 years and residing in Florianópolis are eligible for vaccination by a single dose of $0.5 \mathrm{~mL}$ of Biken's varicella vaccine. This is an Oka vaccine strain produced by Aventis-Pasteur and licensed for children $>1$ year of age. The children were vaccinated in the second year of life. The vaccine was administered subcutaneously.

We focused our attention on four age groups $(<1,1-4$, 5-9 and 10-14 years) of major interest for evaluating a short-term impact of the vaccine. For each age group, annual incidence per 10,000 was calculated separately for the state capital and the rest of the state. A graphical comparison of these incidence rates was followed by calculation of their incidence ratio (IR) for each year of the study period. The IR and its $95 \%$ confidence interval $(95 \% \mathrm{CI})$ were calculated for the periods before (1997-2001) and after (2002-2007) the vaccination in Florianópolis and adjusted for the timetrend effect by linear regression of the IR on the calendar year and period indicator (post- vs. pre-vaccine period), thus separating time-related from vaccine-related effects. Finally, vaccine effectiveness was estimated as one minus the ratio of IR of pre-vaccination period to the IR of postvaccination period (adjusted for time trend).

Stata software was used for all statistical analyses. ${ }^{5}$

Because this study used a secondary data source and did not involve human subjects, it did not require an ethics committee approval.

\section{Results}

During the 1997-2007 period, 135,311 cases of primary varicella infection were reported in the state of Santa Catarina, with $70 \%$ of these among children under 10 years of age. There were no significant differences regarding the distribution of cases by sex. During the period, varicella complications were reported as the cause of death in a 45-year-old patient.

In 2007, the state capital achieved varicella vaccine coverage of 72 and $91 \%$ for children under 5 and 5-6 years of age, respectively. As children aged 7 years or older were not reached by the vaccination program in 2007, and there were no catch-up strategy, the vaccine coverage for the 5-9 age group reduced to $40 \%$ and remained zero for the 10-14 years old.

Figure 1 shows raising trend of varicella incidence among children aged 1-4 years and, to a lesser extent, among children $<1$ year of age in the state of Santa Catarina contrasted with the falling incidence of these age groups in the state capital. On the other hand, older children (5-14 years) did not follow this pattern.

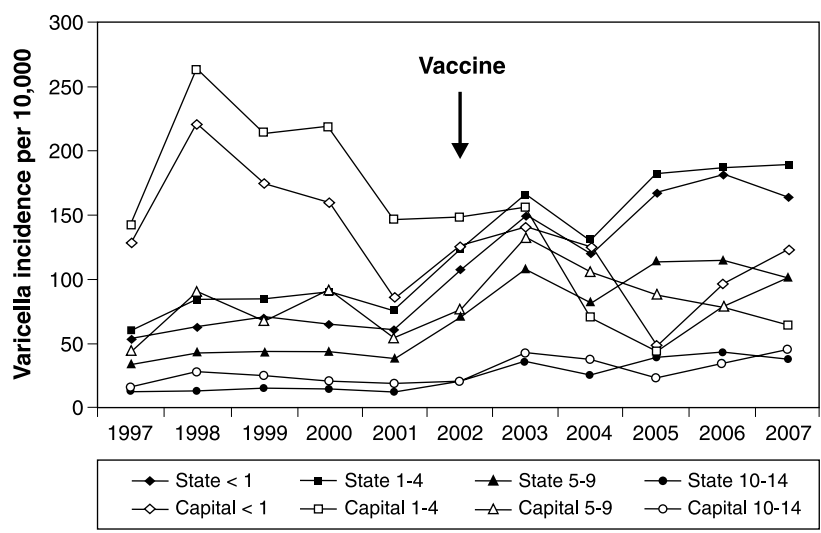

Figure 1 - Incidence of varicella by age group in the state of Santa Catarina and in the capital city (1997-2007)

Statistical analysis of the capital vs. state IR revealed that unadjusted means for all age groups analyzed showed a reduction from pre-vaccination to post-vaccination period, with the largest reduction of $75.5 \%$ (from 2.49 to 0.61 ) for the age group of $1-4$ years (Table 1 ). All age groups, except for the oldest one, had non-overlapping $95 \% \mathrm{CI}$, thus suggesting significant reduction of the IR after the vaccination compared to the pre-vaccination period. However, the reduction remained statistically significant only for the 1-4-year age group after adjusting for time trend (Table 1), and the fraction attributable to the vaccine was estimated at -0.94 . By dividing the latter figure by the baseline value $(-0.94 / 2.49)$, we arrive at a 
$37.75 \%$ (95\%CI 5.62-69.80) percentage reduction from the baseline - a usual estimate of vaccine effectiveness. For other age groups, the effectiveness was not statistically significant and ranged from $27 \%$ in the youngest group to $30 \%$ in the oldest group.

\section{Discussion}

The strongest vaccination effect was observed among children aged 1-4 years in Florianópolis as this group accumulated those vaccinated under 2 years of age. Although the Brazilian Society of Pediatrics recommends vaccinating children against varicella in the second year of life, ${ }^{6}$ occasionally some children in Florianópolis were vaccinated in the first year of life in the case of varicella outbreak control. The absence of vaccine coverage for the children aged 7 years or older in Florianópolis by the end of the period analyzed was clearly limiting the vaccine effectiveness.

It should be borne in mind that the vaccine effectiveness in the range of $27-38 \%$ was achieved in only 6 years of the vaccination program in Florianópolis. The mean vaccine efficacy of one dose of Varivax vaccine (produced by Merck) in preventing all disease was $84.5 \%$, and its efficacy in reducing combined moderate and severe disease reached $97 \%$ (range 86 to $100 \%$ ) for the 12 -year period in the USA.7,8 However, in the 6 years prior to the US vaccination program, the varicella incidence per million population dropped from 2,600 to approximately 1,690 , which is a $35 \%$ reduction. ${ }^{8}$ During the first 6 years of the vaccination program, a $65 \%$ incidence reduction (from 1,690 to 590) indicates an acceleration in an already declining trend, probably due to the beneficial effect of the vaccine. The difference of post-vaccination minus prevaccination incidence reduction in the USA $(65-35=30 \%)$ serves as a rough estimate of the vaccine effectiveness in the first 6 years of the program. The resulting 30\% figure for the USA is adjusted for time trend and falls within the range of 27 to $38 \%$ for the four age groups in Florianópolis. It should be noted that the US vaccination program included a catch-up strategy not implemented in Florianópolis.

A recent review of the US hospitalization rates due to varicella showed an accelerated decline after introduction of mass varicella vaccination. ${ }^{8}$ However, great differences in access to hospital care between the state capital and the rural areas of the state made it difficult to use this indicator of vaccine effectiveness in Brazil.

A considerably higher varicella incidence reported in children $<5$ years in the state capital compared to the rest of the state during the pre-vaccination period may be a consequence of more sensitive epidemiologic surveillance in the capital and higher contact rates in its population due to better availability of child care facilities and higher population density in the state capital.

Our estimates are less precise than the US data because the former are derived from an observational study and a much smaller population. Other limitations of our study include a small number of deaths to evaluate the mortality impact, unknown misclassification rate for the varicella case definition, relatively few time points with large variation between them, and disregard for the indirect vaccine benefits in the neighborhood of the state capital where the vaccination took place. Limited time span for the period after the introduction of varicella vaccine in Florianópolis likely is the reason why we could not confirm the herd immunity effect by means of statistical analyses, although the reduction in the IR ratio for the children aged $5-9$ and $10-14$ years point in that direction

Table 1 - IR of the capital vs. the rest of the state: unadjusted and adjusted time-trend pre-vaccination (PRE-V) vs. post-vaccination (POST-V) estimates (linear regression of IR on calendar year and pre/post-vaccination period)

\begin{tabular}{|c|c|c|c|c|c|}
\hline \multirow[b]{2}{*}{ Age (years) } & \multicolumn{2}{|c|}{ Unadjusted IR (95\%CI) } & \multicolumn{2}{|c|}{ Time-trend adjusted IR ( $95 \% \mathrm{CI})$} & \multirow[b]{2}{*}{$\mathbf{r}^{2 *}$} \\
\hline & PRE-V & POST-V & IR annual change & POST-V vs. PRE-V & \\
\hline$<1$ & $2.46(1.71$ to 3.21$)$ & 0.78 (0.48 to 1.09$)$ & $-0.18(-0.40$ to 0.03$)$ & $-0.67(-2.02$ to 0.68$)$ & 0.82 \\
\hline $1-4$ & 2.49 (2.06 to 2.92$)$ & $0.61(0.27$ to 0.95$)$ & $-0.17(-0.30$ to -0.04$)$ & $-0.94(-1.74 \text { to }-0.14)^{+}$ & 0.94 \\
\hline $5-9$ & $1.68(1.28$ to 2.08$)$ & $1.00(0.10$ to 1.23$)$ & $-0.03(-0.18$ to 0.11$)$ & $-0.48(-1.43$ to 0.46$)$ & 0.58 \\
\hline $10-14$ & 1.59 (1.25 to 1.92$)$ & $1.05(0.75$ to 1.35$)$ & $-0.01(-0.17$ to 0.15$)$ & $-0.48(-1.47$ to 0.51$)$ & 0.44 \\
\hline
\end{tabular}

$95 \% \mathrm{Cl}=95 \%$ confidence interval; IR = incidence ratio; POST-V = post-vaccination; PRE-V = pre-vaccination.

* Coefficient of determination.

$\dagger$ Statistically significant at $p=0.027$. 
(Table 1). The varicella vaccine may have reduced the disease severity and, therefore, has made its diagnosis and report in Florianópolis less frequent, thus leading to the overestimation of the vaccine effectiveness. Finally, as a vaccine efficacy study was beyond the reach of the data available, we decided to use a much simpler design.

A recent review of cost-effectiveness of varicella vaccination programs shows that most countries target only high-risk groups and that this strategy is cost saving. ${ }^{9}$ Universal early-childhood vaccination is also cost saving if indirect costs of production losses are included from a broader society perspective. The USA were the first country to introduce a universal single-dose vaccination against varicella and added a second dose with catchup strategy for older children in $2006 .{ }^{8}$ The single-dose vaccination scheme yielded reductions of disease incidence, hospitalizations and deaths in the range of 57-90, 75-88 and $74-92 \%$, respectively. Australia introduced varicella vaccine by the end of 2005 for all susceptible children at the age of 18 months and $10-13$ years, ${ }^{10}$ but it will take longer to evaluate its effectiveness with some precision. In Latin America, Uruguay is the only country that has implemented a nationwide immunization with varicella vaccine for children, reducing the proportion of hospitalizations due to varicella among children under 15 years of age by $81 \%$ in the first 6 years since the vaccine introduction. ${ }^{11}$

Although varicella vaccine has been effective in reducing the disease burden in the USA, the magnitude of this effect has not been homogenous. For example, some sentinel sites in the USA have seen rising incidence among adolescents 10-19 years of age since the year 2000, resulting in an upward shift of the mean age of varicella infection. ${ }^{12}$ Longer period of observation is needed to know if these are isolated phenomena or a more general trend.

Despite the limitations of our study, it presents the first estimates of the varicella vaccine effectiveness in reducing the disease incidence based on a 6-year universal early childhood vaccination program in Brazil. Longer experience with the program and other study designs (e.g., casecontrol) may be needed to achieve more precise estimates. However, the results so far indicate the incidence reduction compatible with that reported in the USA, thus confirming the viability of such vaccination programs in Brazil.

\section{References}

1. Ministério da Saúde, Brasil. Programa Nacional de Imunizações (PNI). http://portal.saude.gov.br/portal_arquivos/pdf/cries_ indicacoes. Access: 12/03/2009.

2. Valentim J, Sartori AM, de Soárez PC, Amaku M, Azevedo RS, Novaes HM. Cost-effectiveness analysis of universal childhood vaccination against varicella in Brazil. Vaccine. 2008;26:6281-91.

3. Brasil, Ministério da Saúde. DATASUS. População residente - Santa Catarina. http://tabnet.datasus.gov.br/cgi/deftohtm. exe?ibge/cnv/popsc.def. Access: 25/10/2008.

4. Brasil, Ministério da Saúde. Guia de Vigilância Epidemiológica. $6^{a}$ ed. Brasília: Ministério da Saúde; 2005.

5. StataCorp. Stata Statistical Software: Release 9. College Station, TX: StataCorp LP; 2005

6. Bricks LF, Sato HK, Oselka GW. Varicella vaccines and measles, mumps, rubella, and varicella vaccine. J Pediatr (Rio J). 2006;82: S101-8.

7. Seward JF, Marin M, Vázquez M. Varicella vaccine effectiveness in the US vaccination program: a review. J Infect Dis. 2008;197 Suppl 2:S82-9.

8. Marin M, Meissner HC, Seward JF. Varicella prevention in the United States: a review of successes and challenges. Pediatrics. 2008;122:e744-51.

9. Rozenbaum $\mathrm{MH}$, van Hoek $\mathrm{AJ}$, Vegter S, Postma MJ. Costeffectiveness of varicella vaccination programs: an update of the literature. Expert Rev Vaccines. 2008;7:753-82.

10. Macartney KK, BeutelsP, McIntyreP, Burgess MA. Varicella vaccination in Australia. J Paediatr Child Health. 2005;41:544-52.

11. Quian J, Rüttimann R, Romero C, Dall'Orso P, Cerisola A, Breuer $T$, et al. Impact of universal varicella vaccination on 1-year olds in Uruguay: 1997-2005. Arch Dis Child. 2008;93:845-850.

12. Guris D, Jumaan AO, Mascola L, Watson BM, Zhang JX, Chaves SS, et al. Changing varicella epidemiology in active surveillance sites--United States, 1995-2005. J Infect Dis. 2008;197 Suppl 2:S71-5.

Correspondence:

Emil Kupek

Departamento de Saúde Pública, CCS/UFSC

Campus Universitário-Trindade

CEP 88040-900 - Florianópolis, SC - Brazil

Tel.: +55 (48) 3025.6232

Fax: +55 (48) 3721.9542

E-mail: kupek@ccs.ufsc.br 